\title{
DENSE FLAG TRIANGULATIONS OF 3-MANIFOLDS VIA EXTREMAL GRAPH THEORY
}

\author{
MICHAE ADAMASZEK AND JAN HLADKÝ
}

\begin{abstract}
We characterize $f$-vectors of sufficiently large three-dimensional flag Gorenstein* complexes, essentially confirming a conjecture of Gal [Discrete Comput. Geom., 34 (2), 269-284, 2005]. In particular, this characterizes $f$ vectors of large flag triangulations of the 3-sphere. Actually, our main result is more general and describes the structure of closed flag 3-manifolds which have many edges.

Looking at the 1-skeleta of these manifolds we reduce the problem to a certain question in extremal graph theory. We then resolve this question by employing the Supersaturation Theorem of Erdős and Simonovits.
\end{abstract}

\section{INTRODUCTION}

One of the trends in enumerative combinatorics is to classify face numbers of various families of simplicial complexes. In this paper we study flag triangulations of closed 3-manifolds with sufficiently many vertices and high edge density. As a consequence we confirm, for a sufficiently large number of vertices, a conjecture of Gal regarding face vectors of flag triangulations of generalized homology 3-spheres.

If $K$ is a finite simplicial complex and $\sigma \in K$ is a face, we denote by $|\sigma|$ its number of vertices and by $\operatorname{dim} \sigma=|\sigma|-1$ its dimension. The dimension of $K$, $\operatorname{dim} K$, is the maximum over all $\sigma \in K$ of $\operatorname{dim} \sigma$.

The $f$-vector of a simplicial complex $K$ of dimension $d$ is the sequence

$$
\left(f_{-1}, f_{0}, \ldots, f_{d}\right)
$$

where $f_{i}$ is the number of faces of dimension $i$. By convention, we always define $f_{-1}=1$. The $h$-vector of $K$ is the sequence

$$
\left(h_{0}, \ldots, h_{d+1}\right)
$$

determined by the equation 1

$$
\sum_{i=0}^{d+1} h_{i} x^{d+1-i}=\sum_{i=-1}^{d} f_{i}(x-1)^{d-i} .
$$

Received by the editors November 29, 2012 and, in revised form, April 11, 2013.

2010 Mathematics Subject Classification. Primary 05E45; Secondary 05A15.

Key words and phrases. f-vector, simplicial complex, Gal's conjecture, flag triangulations of 3-manifolds.

The research of the first author was carried out while he was a member of the Centre for Discrete Mathematics and its Applications (DIMAP), supported by the EPSRC award EP/D063191/1.

The second author is an EPSRC Research Fellow.

${ }^{1}$ Note that we consistently use $d$ for the dimension of $K$, rather than the cardinality of its largest face. Hence the indices and exponents in most formulae are shifted by 1 compared to what they usually look like. 
Of course the $f$-vector and the $h$-vector determine one another and carry the same information, but the $h$-vector often enjoys better combinatorial properties, the Dehn-Sommerville equation (44) below being one example. Note that $h_{0}=1$.

Next we introduce the class of Gorenstein* and Eulerian complexes. The reader not interested in this level of generality can equally well think about simplicial complexes which triangulate a standard sphere. Recall that if $\sigma \in K$ is a face, then the link of $\sigma$ in $K$, denoted $\operatorname{lk}_{K} \sigma$, is the subcomplex $\{\tau \in K \mid \tau \cap \sigma=\emptyset, \tau \cup \sigma \in K\}$.

A simplicial complex $K$ of dimension $d$ is a generalized homology sphere (or Gorenstein ${ }^{*}$ complex) if for every face $\sigma \in K$ the homology of $\operatorname{lk}_{K} \sigma$ is the same as the homology of a sphere of dimension $d-|\sigma|$. In particular, when $\sigma=\emptyset$, this means that $K$ itself has the homology of a $d$-sphere. We are going to use the short name ' $d$-GHS'. A simplicial complex $K$ of dimension $d$ is Eulerian if for every face $\sigma \in K$ the Euler characteristic of $\mathrm{lk}_{K} \sigma$ is the same as that of a sphere of dimension $d-|\sigma|$.

Any triangulation of the standard $d$-sphere is a $d$-GHS and every $d$-GHS is Eulerian. More generally, if $K$ is a triangulation of a closed topological manifold and $\sigma \neq \emptyset$ is a face of $K$, then $\operatorname{lk}_{K} \sigma$ is a $(d-|\sigma|)$-GHS. By the Poincaré duality the Euler characteristic of an odd-dimensional closed manifold is 0 , hence every such manifold is Eulerian. (A closed manifold means a compact manifold without boundary.)

Any Eulerian complex of dimension $d$ satisfies the classical Dehn-Sommerville equations

$$
h_{i}=h_{d+1-i},
$$

and, following Gal Gal05, one can encode the coefficients $h_{i}$ in a shorter, integervalued $\gamma$-vector

$$
\left(\gamma_{0}, \ldots, \gamma_{\left\lfloor\frac{d+1}{2}\right\rfloor}\right)
$$

determined by the equation

$$
\sum_{i=0}^{d+1} h_{i} x^{i}=\sum_{i=0}^{\left\lfloor\frac{d+1}{2}\right\rfloor} \gamma_{i} x^{i}(x+1)^{d+1-2 i} .
$$

We always have $\gamma_{0}=1$.

The classification of $h$ - (or $f-, \gamma$-) vectors of generalized homology spheres is of great interest in the field. The complete classification is predicted by the celebrated $g$-conjecture of McMullen [McM71]. In this work we pick up a related but somewhat different line of research started by Gal, who investigated these parameters for the restricted family of flag complexes.

A simplicial complex is a flag if all its minimal non-faces have dimension 1 or, equivalently, if it is the clique complex of its 1-skeleton. The latter means that faces of $K$ correspond to cliques in $K^{(1)}$, the graph which is the one-dimensional skeleton of $K$. For flag generalized homology spheres the $\gamma$-vector is the most efficient and interesting parameter. The major conjecture of Gal [Gal05, Conj. 2.1.7], which states that the $\gamma$-vector of a flag $d$-GHS is non-negative, is known to hold for $d \leq 4$ Gal05, Cor.2.2.3]. For any flag $(2 d-1)$-GHS this conjecture is a strengthening of the famous Charney-Davis conjecture CD95. On the other hand, Gal's conjecture itself has a stronger version which states that the $\gamma$-vector of a flag $d$-GHS is an 
$f$-vector of some flag complex [NP11. See [NPT11] and the references therein for progress in that area.

If $K$ and $L$ are two simplicial complexes with disjoint vertex sets, then their join $K * L$ is a simplicial complex with vertex set $V(K) \cup V(L)$ whose faces are all unions $\tau \cup \sigma$ for $\tau \in K, \sigma \in L$. It is a standard fact that $S^{k} * S^{l}=S^{k+l+1}$ for triangulated spheres $S^{k}, S^{l}$ with $k, l \geq-1$.

Following Murai and Nevo [MN], let $\Lambda_{d}$ denote the set of all $\gamma$-vectors of flag $d$-GHSs. When $d=1,2$ the $(k+4)$-gon or its join with the two-point sphere $S^{0}$ are simplicial $d$-spheres with $\gamma$-vector $(1, k)$ for any integer $k \geq 0$, and by the previous discussion these exhaust $\Lambda_{1}$ and $\Lambda_{2}$, i.e., we have

$$
\Lambda_{1}=\Lambda_{2}=\left\{(1, k) \in \mathbb{Z}^{2}: k \geq 0\right\} .
$$

Gal [Gal05, Cor. 3.1.7] proved that $\gamma_{2} \leq \gamma_{1}^{2} / 4$ must hold for any $\gamma$-vector $\left(1, \gamma_{1}, \gamma_{2}\right)$ in $\Lambda_{3}$ or $\Lambda_{4}$, and a simple join construction [MN, Thm. 5.1.ii] shows that this is tight in dimension 4 ; that is,

$$
\Lambda_{4}=\left\{\left(1, \gamma_{1}, \gamma_{2}\right) \in \mathbb{Z}^{3}: \gamma_{2} \leq \frac{\gamma_{1}^{2}}{4}, \quad \gamma_{1}, \gamma_{2} \geq 0\right\}
$$

Going back to dimension 3, Gal [Gal05, Thm. 3.2.1] showed that

$$
\begin{aligned}
& \Lambda_{3} \supseteq\left\{\left(1, \gamma_{1}, \gamma_{2}\right) \in \mathbb{Z}^{3}: \gamma_{2} \leq \frac{\left(\gamma_{1}-1\right)^{2}}{4}, \quad \gamma_{1}, \gamma_{2} \geq 0\right\} \\
& \cup\left\{(1, k+l, k l) \in \mathbb{Z}^{3}: k, l \geq 0\right\} .
\end{aligned}
$$

The elements of the first set can be realized as $\gamma$-vectors of some appropriate iterated edge subdivisions of the boundary of the cross-polytope. The elements of the second kind are the $\gamma$-vectors of a join of a $(k+4)$-gon with an $(l+4)$-gon.

Gal then conjectured that the inclusion (7) is in fact an equality. Since the $\gamma$ vector of a flag 3-GHS is non-negative, the stronger version of that conjecture is the following (see [Gal05, Con. 3.2.2] or [MN, Conj. 5.2]).

Conjecture 1.1. If $\left(1, \gamma_{1}, \gamma_{2}\right)$ is the $\gamma$-vector of a flag 3-GHS $K$ and $\gamma_{2}>\frac{\left(\gamma_{1}-1\right)^{2}}{4}$, then $K$ is a join of two polygons.

Also, note that the two constructions which show the inclusion (77) are flag triangulations of the 3 -sphere. Thus - if true - Conjecture 1.1 provides a characterization of $\gamma$-vectors (or $f$-vectors) of flag triangulations of the 3 -sphere. Even this special case of the characterization of $\gamma$-vectors of flag triangulations of the 3 -sphere is open. The conjecture was verified for order complexes of posets [MN].

To make the following discussion more concrete, suppose that $K$ is an Eulerian complex of dimension 3 with face numbers $\left(1, f_{0}, f_{1}, f_{2}, f_{3}\right)$. Then the DehnSommerville relations translate into

$$
f_{2}=2\left(f_{1}-f_{0}\right), \quad f_{3}=f_{1}-f_{0} .
$$

Moreover, we find

$$
\gamma_{1}=f_{0}-8, \quad \gamma_{2}=f_{1}-5 f_{0}+16
$$

and the conditions $\left(\gamma_{1}-1\right)^{2} / 4<\gamma_{2} \leq \gamma_{1}^{2} / 4$ are equivalent to

$$
\frac{1}{4}\left(f_{0}^{2}+2 f_{0}+17\right)<f_{1} \leq \frac{1}{4} f_{0}^{2}+f_{0} .
$$


Our results. Below is the main result of the paper. It determines the structure of closed flag 3-manifolds which have many edges.

Theorem 1.2. There exists a number $n_{0}$ such that the following holds. If $M$ is a flag triangulation of a closed 3-manifold with $f_{0} \geq n_{0}$ vertices, $f_{1}$ edges, and such that $f_{1}>\frac{1}{4}\left(f_{0}^{2}+2 f_{0}+17\right)$, then $M$ is a join of two polygons (and, in particular, it is homeomorphic to $\left.S^{3}\right)$.

Theorem 1.2 resolves Conjecture 1.1 affirmatively for flag complexes with sufficiently many vertices because every 3 -GHS is a closed manifold (see Remark 1.9). In other words, the inclusion (77) is an equality except for, perhaps, a finite number of elements.

Below, we prepare tools for our proof of Theorem 1.2. We shall reduce Theorem 1.2 to a certain statement in extremal graph theory (Theorem 1.6).

Given a graph $G$ and a vertex $v \in V(G)$ we write $N_{v}$ for the neighborhood of $v$; that is, $\{w \in V(G): v w \in E(G)\}$. If $W \subseteq V(G)$, then $G[W]$ is the subgraph of $G$ induced by $W$. The length of a path in a graph is its number of vertices; this is one more than the standard common definition of length but more convenient for our purposes.

Definition 1.3. If $G$ is a graph and $\sigma$ is a clique in $G$, then define the link of $\sigma$ in $G$ as

$$
\operatorname{lk}_{G} \sigma=G\left[\bigcap_{v \in \sigma} N_{v}\right] .
$$

That is, $\operatorname{lk}_{G} \sigma$ is the subgraph of $G$ induced by the vertices which are not in $\sigma$ but which are adjacent to every vertex of $\sigma$.

Definition 1.3 is designed so that it is compatible with the topological notion of links in flag complexes. For each flag complex $K$ we have $\operatorname{lk}_{K^{(1)}} \sigma=\left(\mathrm{lk}_{K} \sigma\right)^{(1)}$, where on the left-hand side we use the link of Definition 1.3 and on the right-hand side the link is understood in the simplicial sense.

Let us define the class of graphs which arise in our setting.

Definition 1.4. A graph $G$ with $n$ vertices and $m$ edges is fascinating if it satisfies the following conditions:

a) $G$ contains exactly $2(m-n)$ triangles.

b) For every edge $e$ in $G$ the link $\operatorname{lk}_{G} e$ is a cycle of length at least 4 .

c) For every triangle $t$ in $G$ the link $\mathrm{lk}_{G} t$ is the discrete graph with 2 vertices and no edges.

d) For every vertex $v$ in $G$ the link $\operatorname{lk}_{G} v$ is a connected, planar graph whose every face (including the unbounded one) is a triangle. In particular - by Kuratowski's Theorem - it does not contain the complete bipartite graph $K_{3,3}$ as a subgraph.

Further, $\mathrm{lk}_{G} v$ contains at least 6 vertices.

Our reduction is based on the next observation.

Lemma 1.5. If $M$ is a closed flag 3-manifold, then the 1-skeleton of $M$ is fascinating.

Proof. Let $G=M^{(1)}$. Condition a) follows since $M$ is Eulerian, and so it satisfies (8). Parts b)-d) are consequences of the fact that $\mathrm{lk}_{M} t, \mathrm{lk}_{M} e, \mathrm{lk}_{M} v$ are flag triangulations of, respectively, $S^{0}, S^{1}$ and $S^{2}$. A known fact that a flag triangulation of 
$S^{j}$ requires at least $2(j+1)$ vertices [Gal05, Lem.2.1.14] proves that the links must be sufficiently large.

The graph join of graphs $G$ and $H$, which we will denote $G * H$, is the disjoint union of $G$ and $H$ together with all the edges between $V(G)$ and $V(H)$. For any simplicial complexes $K$ and $L$ we have $(K * L)^{(1)}=K^{(1)} * L^{(1)}$, where on the left-hand side we use the simplicial join.

By Lemma 1.5 we get that Theorem 1.2 is a consequence of the following result.

Theorem 1.6. There exists a number $n_{0}$ such that the following holds. Suppose $G$ is a fascinating graph with $n \geq n_{0}$ vertices, $m$ edges and $m>\frac{1}{4}\left(n^{2}+2 n+17\right)$. Then $G$ is a join of two cycles.

The rest of the paper is concerned with the proof of this theorem. The strategy is outlined at the beginning of the next section.

Remark 1.7. Along the way we will also see that the result is tight in the following sense: There exist flag 3 -spheres with arbitrarily large $f_{0}$ and with exactly

$$
f_{1}=\frac{1}{4}\left(f_{0}^{2}+2 f_{0}+17\right)
$$

edges, which are not a join of two cycles. Moreover, we will classify those boundary cases: Any fascinating graph $G$ with $n \geq n_{0}$ vertices and exactly $m=\frac{1}{4}\left(n^{2}+2 n+17\right)$ edges is one of the graphs in Figure 2 in Section 4.

Remark 1.8. Theorem 1.2 implies that for $f_{0} \geq n_{0}$ every closed flag 3 -manifold satisfies $f_{1} \leq \frac{1}{4} f_{0}^{2}+f_{0}$ (or, equivalently, $\gamma_{2} \leq \frac{1}{4} \gamma_{1}^{2}$ ). This result in fact holds for all values of $f_{0}$ by the same proof that works for 3-GHSs in Gal05.

Remark 1.9. In dimensions $d=0,1,2$ the classes of (flag) $d$-spheres and $d$-GHS coincide and in dimension $d=3$ every 3 -GHS is a closed, connected manifold. To see this, first note that it is an easy consequence of the definition that if $L$ is a $d$-GHS and $\sigma \in L$, then $\operatorname{lk}_{L} \sigma$ is a $(d-|\sigma|)$-GHS. Now the only 0 -complex with the homology of $S^{0}$ is $S^{0}$ itself. As for $d=1$, observe that in a 1-GHS all vertex links are the two-point space, so a 1-GHS is a disjoint union of cycles, of which only a single cycle has the homology of $S^{1}$. In a 2-GHS the link of every vertex is the sphere $S^{1}$, so a 2-GHS is a closed surface, and of all surfaces only $S^{2}$ has the correct homology. Finally, it means that in a 3-GHS all face links are homeomorphic to spheres of appropriate dimensions, so a 3 -GHS is a closed manifold.

\section{Proof of Theorem 1.6}

The main idea behind our approach is that $G$ has a lot of edges (more than $n^{2} / 4$ ), but relatively few triangles - just $\Theta\left(n^{2}\right)$. Graphs with this edge density must have many more triangles, namely $\Theta\left(n^{3}\right)$, unless they look very "similar", in some sense, to the complete bipartite graph $K_{n / 2, n / 2}$. This phenomenon is called supersaturation and is one of the basic principles of extremal (hyper)graph theory with fundamental applications to areas like additive combinatorics or property testing in computer science. In our setting the additional properties of $G$ coming from Definition 1.4 can be used to refine the similarity to $K_{n / 2, n / 2}$ to determine the structure of $G$ exactly. This is a relatively standard approach in Extremal Graph Theory, called the Stability method, and was introduced by Simonovits Sim68. However, our proof is somewhat more complex than most of the applications of the 
Stability method to problems in extremal graph theory. Indeed, in these problems one usually tries to determine exactly the structure of a unique extremal graph, while here we are dealing with joins of two cycles whose lengths can vary, i.e., graphs with a somewhat looser structure.

Here is a more detailed outline of the proof. Mantel's Theorem (which is a special case of Turán's Theorem) asserts that the complete balanced bipartite graph $K_{\lfloor h / 2\rfloor,\lceil h / 2\rceil}$ is the unique maximizer of the number edges among all triangle-free graphs on $h$ vertices. Note that this graph has $\left\lfloor h^{2} / 4\right\rfloor$ edges. The graph $K_{\lfloor h / 2\rfloor,\lceil h / 2\rceil}$ is stable for this extremal problem in the following sense: if $H$ is a graph on $h$ vertices with at least $h^{2} / 4$ edges and containing only $o\left(h^{3}\right)$ triangles, it must be "very similar" (the precise meaning appears in Theorem 2.2 ) to $K_{\lfloor h / 2\rfloor,\lceil h / 2\rceil}$. These conditions are satisfied for the fascinating graph $G$ of Theorem 1.6. By exploiting other properties of $G$ we will be able to show that $G$ is close to being a join of two cycles in the sense of the next definition.

Definition 2.1. A fascinating graph $G$ is called $t$-joinlike if there is a partition $V(G)=C_{1} \sqcup C_{2} \sqcup X$ where

- the graphs $G\left[C_{i}\right]$ are cycles,

- there are edges $e_{i} \in G\left[C_{i}\right]$ such that $\operatorname{lk}_{G} e_{i}=G\left[C_{3-i}\right]$,

- $|X|=t$.

The vertices of $X$ are called exceptional.

Note that a 0 -joinlike fascinating graph is a join of two cycles $G\left[C_{1}\right] * G\left[C_{2}\right]$. At the end of this section we will establish that $G$ must be $t$-joinlike for $t=0,1$ or 2 with some extra conditions satisfied by the exceptional vertices.

Observe that the balanced join of two cycles of lengths $\approx \frac{n}{2}$ has $\approx \frac{n^{2}}{4}+n$ edges (and joins of cycles of unbalanced lengths have even less edges), so our graph $G$ is only allowed to "lose" $\approx \frac{n}{2}$ edges with respect to that number before it violates the bound of Theorem 1.6. In many cases, however, we will be able to show that a 2-joinlike graph loses a lot more just by counting the edges missing in the sparse planar links of exceptional vertices (Definition 1.4 d)).

This leaves us with just a handful of possible scenarios considered in Section 4 . Those are the difficult ones, in the sense that the graphs $G$ approach, and in fact even reach, the bound $m=\frac{1}{4}\left(n^{2}+2 n+17\right)$. That means we can no longer use rough estimates. We then have to examine the structure of $G$ more closely. This is the part where the examples shown in Remark 1.7 show up.

Let $e(H)=|E(H)|$, and we write $e(H[A, B])$ (resp. $\bar{e}(H[A, B]))$ for the number of edges (resp. non-edges) crossing between two disjoint vertex sets $A, B \subseteq V(H)$.

Let us now state a theorem of Erdős and Simonovits [ES83, Theorem 3], tailored to our needs 2 As said above, this version of the Supersaturation Theorem gives an approximate structure in graphs with edge density at least $\frac{1}{2}$ which contain subcubically many triangles in the order of the graph 3

\footnotetext{
${ }^{2}$ These days, similar theorems are typically proven with the help of the Szemerédi Regularity Lemma [Sze78; see for example [KS96. Theorem 2.9]. Even though the Regularity Lemma was already alive by the time of the publishing of [ES83] the theory was too juvenile to yield such a statement back then. Therefore some alternative "sieve" arguments were used instead.

${ }^{3}$ The general version of the Supersaturation Theorem deals with (hyper)graphs containing a small number of copies of a fixed (hyper)graph $F$.
} 
Theorem 2.2. For every $\varepsilon>0$ there exists $\delta>0$ such that the following holds. Let $H$ be an $h$-vertex graph with at least $h^{2} / 4$ edges and containing at most $\delta h^{3}$ triangles. Then there exists a partition $V(H)=A_{1} \sqcup A_{2}$, with ||$A_{1}|-| A_{2}|| \leq 1$, such that

$$
e\left(H\left[A_{1}\right]\right)+e\left(H\left[A_{2}\right]\right)+\bar{e}\left(H\left[A_{1}, A_{2}\right]\right) \leq \varepsilon h^{2} .
$$

To obtain the above statement set $\mathcal{L}$ to the one-element family consisting of just a triangle in [ES83, Theorem 3].

We can now proceed with the proof of Theorem 1.6. Let $0<\gamma \ll 1, \alpha<\gamma / 1000$ and $\varepsilon<\alpha \gamma$ be fixed. Let $\delta$ be given by Theorem 2.2 for input parameter $\varepsilon$. Let $n_{0}$ be sufficiently large. Suppose that $G$ is the graph as in Theorem 1.6. Definition [1.4a) gives us that $G$ has $2(e(G)-n)<n^{2}<\delta n^{3}$ triangles. Therefore, Theorem 2.2 applies with parameters $\delta$ and $\varepsilon$. Let $A_{1} \sqcup A_{2}$ be the partition of $V(G)$ from Theorem 2.2 .

Let us fix additional notation. Given a vertex $v$ and a set of vertices $X$ we write

$$
\operatorname{deg}(v, X)=\left|N_{v} \cap X\right| .
$$

Define the following vertex sets for $i=1,2$ :

$$
\begin{aligned}
B_{i} & =\left\{v \in A_{i}: \operatorname{deg}\left(v, A_{3-i}\right) \geq \frac{n}{2}-\gamma n\right\}, \\
W_{i} & =\left\{v \in A_{i} \backslash B_{i}: \operatorname{deg}\left(v, B_{i}\right) \geq \frac{n}{2}-\gamma n\right\}, \\
X_{i} & =\left(A_{i} \backslash B_{i}\right) \backslash W_{i} .
\end{aligned}
$$

Claim 1. We have $\left|A_{i} \backslash B_{i}\right| \leq \alpha n$ for $i=1,2$. In particular, $\left|W_{i}\right|,\left|X_{i}\right|<\alpha n$ and $\left|B_{i}\right| \geq \frac{n}{2}-\alpha n$.

Proof. By definition every vertex of $A_{i} \backslash B_{i}$ has at least $\gamma n-1$ non-edges to $A_{3-i}$. If we had $\left|A_{i} \backslash B_{i}\right|>\alpha n$, then

$$
\bar{e}\left(G\left[A_{1}, A_{2}\right]\right) \geq\left|A_{i} \backslash B_{i}\right| \cdot(\gamma n-1) \geq \alpha \gamma n^{2}-\alpha n>\varepsilon n^{2},
$$

contrary to the choice of $A_{1}$ and $A_{2}$.

Now define the partition $V(G)=S_{1} \sqcup S_{2} \sqcup X$ as follows:

$$
\begin{aligned}
S_{i} & =B_{i} \cup W_{3-i}, \\
X & =X_{1} \cup X_{2} .
\end{aligned}
$$

Observe that $\frac{n}{2}-\alpha n \leq\left|S_{i}\right| \leq \frac{n}{2}+\alpha n$ and $|X| \leq 2 \alpha n$. Denote $x=|X|$. It is our goal to show that $X=\emptyset$, that $S_{1}$ and $S_{2}$ induce cycles, and that the bipartite graph between $S_{1}$ and $S_{2}$ is complete.

Claim 2. For $i=1,2$ and for every vertex $v \in S_{i}$ we have $\operatorname{deg}\left(v, S_{3-i}\right) \geq \frac{n}{2}-2 \gamma n$.

Proof. If $v \in B_{i}$, then $v$ has at least $\frac{n}{2}-\gamma n$ neighbors in $A_{3-i}$, and by Claim 11 at least $\frac{n}{2}-2 \gamma n$ of them hit $B_{3-i}$. If $v \in W_{3-i}$, then $v$ has at least $\frac{n}{2}-\gamma n$ neighbors in $B_{3-i}$.

Claim 3. For $i=1,2$ and for every vertex $v \in S_{i}$ we have $\operatorname{deg}\left(v, S_{i}\right) \leq 2$. Consequently, $e\left(G\left[S_{1}\right]\right)+e\left(G\left[S_{2}\right]\right) \leq n$. Moreover, $G\left[S_{i}\right]$ is triangle-free. 
Proof. Suppose a vertex $v \in S_{i}$ has three neighbors $u_{1}, u_{2}, u_{3} \in S_{i}$. By Claim 2 we have

$$
\left|N_{v} \cap N_{u_{1}} \cap N_{u_{2}} \cap N_{u_{3}} \cap S_{3-i}\right| \geq \frac{n}{2}-13 \gamma n \geq 3 .
$$

This implies that $\operatorname{lk}_{G} v$ contains a copy of $K_{3,3}$ (with $u_{1}, u_{2}, u_{3}$ on one side and the other being in $S_{3-i}$ ), which is a contradiction to Definition 1.4d).

The proof of the last statement is similar: if $t$ is a triangle in $G\left[S_{i}\right]$, then $\operatorname{lk}_{G} t$ contains most of $S_{3-i}$, so $G$ fails Definition 1.4 c).

Claim 4. If $v \in X$, then $\operatorname{deg}\left(v, S_{i}\right) \leq \frac{n}{2}-\frac{2}{3} \gamma n$ for $i=1,2$.

Proof. By definition every vertex $v \in X$ satisfies $\operatorname{deg}\left(v, B_{i}\right) \leq \frac{n}{2}-\gamma n$ for $i=1,2$. Therefore

$$
\operatorname{deg}\left(v, S_{i}\right) \leq \operatorname{deg}\left(v, B_{i}\right)+\left|W_{3-i}\right| \leq \frac{n}{2}-\gamma n+\alpha n \leq \frac{n}{2}-\frac{2}{3} \gamma n .
$$

We call a vertex $v \in X$ poor if $\operatorname{deg}\left(v, S_{1}\right) \geq 3$ and $\operatorname{deg}\left(v, S_{2}\right) \geq 3$. Let $P \subseteq X$ be the set of poor vertices. Choose a partition $X \backslash P=T_{1} \sqcup T_{2}$ such that the vertices $v \in T_{i}$ satisfy $\operatorname{deg}\left(v, S_{i}\right) \leq 2$ for $i=1,2$. Let $p=|P|$.

Claim 5. If $v \in X \backslash P$, then $\operatorname{deg}\left(v, S_{1} \cup S_{2}\right) \leq \frac{n}{2}-\frac{1}{2} \gamma n$.

Proof. This is obvious from Claim 4 .

Claim 6. If $v \in P$, then $\operatorname{deg}\left(v, S_{i}\right) \leq 12 \gamma n$ for $i=1,2$.

Proof. Suppose the contrary, and without loss of generality let $\operatorname{deg}\left(v, S_{2}\right)>12 \gamma n$. Let $u_{1}, u_{2}, u_{3} \in N_{v} \cap S_{1}$ be three different vertices. By Claim 2 the set $N_{u_{1}} \cap N_{u_{2}} \cap$ $N_{u_{3}} \cap S_{2}$ has at least $\frac{n}{2}-10 \gamma n$ vertices; therefore $N_{v}$ hits at least $\gamma n$ of them. In particular, $G\left[N_{v}\right]$ contains a $K_{3,3}$, a contradiction.

We can now plug in the bounds from the claims above to count the number of edges in $G$ to obtain the following bound:

$$
\begin{aligned}
\frac{1}{4} n^{2}+\frac{1}{2} n+\frac{17}{4}<e(G) & \leq e\left(G\left[S_{1}, S_{2}\right]\right)+e\left(G\left[S_{1}\right]\right)+e\left(G\left[S_{2}\right]\right)+e\left(G\left[P, S_{1} \cup S_{2}\right]\right) \\
& +e\left(G\left[X \backslash P, S_{1} \cup S_{2}\right]\right)+\left(\begin{array}{c}
|X| \\
2
\end{array}\right) \\
\leq & \left(\frac{n-x}{2}\right)^{2}+n+24 p \gamma n+(x-p)\left(\frac{n}{2}-\frac{1}{2} \gamma n\right)+\frac{x^{2}}{2} .
\end{aligned}
$$

This is equivalent to

$$
x\left(\frac{\gamma n}{2}-\frac{3}{4} x\right)+\frac{p n}{2}(1-49 \gamma)+\frac{17}{4}<\frac{n}{2} .
$$

Since $x \leq 2 \alpha n<\frac{1}{3} \gamma n$, we have $\frac{\gamma n}{2}-\frac{3}{4} x>\frac{\gamma n}{4}$, and the last inequality implies

$$
\frac{x \gamma n}{4}+\frac{p n}{2}(1-49 \gamma)+\frac{17}{4}<\frac{n}{2} \text {. }
$$

It follows that

$$
\begin{aligned}
& x<\frac{2}{\gamma} \text { and } \\
& p<\frac{1}{1-49 \gamma}<1.5 .
\end{aligned}
$$


In particular, we can only have $p=0$ or $p=1$.

Let $K_{i}=S_{i} \cup T_{i}$ for $i=1,2$. Note that

$$
\frac{n}{2}-\alpha n \leq\left|K_{i}\right| \leq \frac{n}{2}+\alpha n+x \leq \frac{n}{2}+2 \alpha n .
$$

Let $b=\bar{e}\left(G\left[K_{1}, K_{2}\right]\right)$ be the number of missing edges between $K_{1}$ and $K_{2}$. The following bound follows directly from Claim 3. the definition of $T_{i}$ and (13).

Claim 7. For each $v \in K_{i}$ we have that $\operatorname{deg}\left(v, K_{i}\right) \leq\left|T_{i}\right|+2 \leq x+2 \leq \frac{4}{\gamma}$.

Claim 8. For $i=1,2$ and each set $Y \subseteq S_{i},|Y| \leq \frac{n}{8}$ we have that $G\left[S_{i} \backslash Y\right]$ contains at least one edge. In particular, $G\left[S_{i}\right]$ contains at least one edge.

Proof. Suppose the claim does not hold for example for $i=1$ and some set $Y \subseteq S_{1}$. Let $t_{i}$ be the number of triangles in $G$ with at least two vertices in $K_{i}$.

If $T_{2} \neq \emptyset$, then let us consider an arbitrary fixed vertex $v \in T_{2}$. By Claim 7 inside $K_{2}$ there are at most $\operatorname{deg}\left(v, K_{2}\right)^{2} \leq 16 / \gamma^{2}$ triangles touching $v$. We further see that there are at most $\operatorname{deg}\left(v, K_{2}\right)\left|K_{1}\right| \leq 4 n / \gamma$ triangles through $v$ with two vertices in $K_{2}$ (one of them being $v$ ) and one vertex in $K_{1}$. Summing over all $v \in T_{2}$ we get that the number of triangles touching $T_{2}$ with at least two vertices in $K_{2}$ is at most $\left|T_{2}\right| \times\left(\frac{16}{\gamma^{2}}+\frac{4 n}{\gamma}\right) \leq \frac{17 n}{\gamma^{2}}$.

To bound $t_{2}$ it only remains to add triangles whose two vertices are in $S_{2}$ and the third is in $K_{1}$ (by Claim 3 there are no triangles entirely inside $S_{2}$ ). By Claim 3 we have

$$
e\left(G\left[S_{2}\right]\right) \leq\left|S_{2}\right| \leq \frac{11 n}{20} .
$$

Since each edge in $S_{2}$ can be extended in at most $\left|K_{1}\right| \leq \frac{11 n}{20}$ ways to such a triangle we get that

$$
t_{2} \leq \frac{17 n}{\gamma^{2}}+\frac{11 n}{20} \cdot \frac{11 n}{20} \leq \frac{122 n^{2}}{400} .
$$

To bound the number $t_{1}$ of triangles with at least two vertices inside $K_{1}$ we proceed similarly, except that the fact $e\left(G\left[S_{1} \backslash Y\right]\right)=0$ allows us to strengthen the counterpart of (15) to $e\left(G\left[S_{1}\right]\right) \leq 2|Y| \leq \frac{n}{4}$. Consequently,

$$
t_{1} \leq \frac{17 n}{\gamma^{2}}+\frac{n}{4} \cdot \frac{11 n}{20} \leq \frac{3 n^{2}}{20} .
$$

Finally, the number $t_{P}$ of triangles passing through the (at most one) poor vertex in $P$ satisfies $t_{P} \leq(24 \gamma n+x)^{2}<700 \gamma^{2} n^{2}<0.01 n^{2}$ by Claim 6 .

We get that the total number of triangles is $t_{1}+t_{2}+t_{P}<0.47 n^{2}<2(e(G)-n)$, a contradiction to Definition 1.4a).

Next, we claim that there are no poor vertices.

Claim 9. We have $p=0$.

Proof. Suppose that $p=1$ and let $P=\{q\}$. Employing Claim 3 and the definition of $T_{1}, T_{2}$ we get

$$
\begin{aligned}
e\left(G\left[K_{1} \cup K_{2}\right]\right) & \leq\left(\frac{n-1}{2}\right)^{2}-b+\sum_{i=1,2}\left(\left|S_{i}\right|+2\left|T_{i}\right|+\left(\begin{array}{c}
\left|T_{i}\right| \\
2
\end{array}\right)\right) \\
& \stackrel{130}{\leq}\left(\frac{n-1}{2}\right)^{2}-b+n+C
\end{aligned}
$$


where $C$ depends only on $\gamma$. By Claim [6] we then have the following estimate:

$$
\frac{1}{4} n^{2}+\frac{1}{2} n+\frac{17}{4}<e(G) \leq\left(\frac{n-1}{2}\right)^{2}-b+n+25 \gamma n .
$$

This implies

$$
b \leq 25 \gamma n \text {. }
$$

Consider any edge $e \in G\left[S_{1}\right]$. The link $\mathrm{lk}_{G} e$ is a cycle $C$ which contains, by Claim 2, at least $\frac{n}{2}-6 \gamma n$ vertices of $S_{2}$ and, by Claim 3, does not pass through $S_{1}$. The number of vertices in which $C$ can exit $S_{2}$ is bounded from above by $2(x+1)$. Eliminating the vertices of $C$ which are adjacent (in the graph $G$ ) to $T_{2}$ (at most $2 x$ ) or to $q$ (at most $12 \gamma n$ by Claim [6), we find that $G\left[S_{2}\right]$ contains at least $\frac{1}{2}\left(\frac{n}{2}-30 \gamma n\right)$ vertex-disjoint edges $e^{\prime}=u^{\prime} v^{\prime}$ which satisfy $V\left(\operatorname{lk}_{G} e^{\prime}\right) \subseteq K_{1}$.

We claim that for at least one such edge $e^{\prime}=u^{\prime} v^{\prime}$ we have $K_{1} \subseteq N_{u^{\prime}} \cap N_{v^{\prime}}$. Indeed, each edge $e^{\prime}$ for which this does not hold is incident with at least one nonedge in $G\left[K_{1}, K_{2}\right]$, and thus otherwise we would get at least $\frac{1}{2}\left(\frac{n}{2}-30 \gamma n\right)$ non-edges in $G\left[K_{1}, K_{2}\right]$, a contradiction to (17).

Let us fix an edge $e^{\prime}$ as above. We now have that $\operatorname{lk}_{G} e^{\prime}=G\left[K_{1}\right]$, and therefore $G\left[K_{1}\right]$ is a cycle. A symmetric argument starting with an appropriate edge $e^{\prime \prime} \in$ $G\left[K_{1}\right]$ for which $\mathrm{lk}_{G} e^{\prime \prime}=G\left[K_{2}\right]$ shows that $G\left[K_{2}\right]$ is a cycle as well.

We now see that $G$, with the decomposition $V(G)=K_{1} \sqcup K_{2} \sqcup\{q\}$, is 1-joinlike in the sense of Definition 2.1. We shall however later in Proposition 4.2 show that this leads to a contradiction.

For the remaining part we can therefore assume $P=\emptyset$. Our short-term goal for now is to prove that $G$ is 0 -, 1 - or 2 -joinlike. The same way we derived (16) we get that

$$
\frac{1}{4} n^{2}+\frac{1}{2} n+\frac{17}{4}<e(G) \leq\left(\frac{n}{2}\right)^{2}-b+n+\frac{4}{\gamma^{2}} .
$$

This implies

$$
b<\frac{n}{2}+\frac{4}{\gamma^{2}}-\frac{17}{4}<0.51 n .
$$

Let $E_{i}$ be the set containing $T_{i}$ and all the neighbors in $S_{i}$ of the vertices in $T_{i}$. By definition of $T_{i}$ we have $\left|E_{i}\right| \leq 3 x$. Note that $K_{i} \backslash E_{i}=S_{i} \backslash E_{i}$, and for any vertex $v \in K_{i} \backslash E_{i}$ we have $\operatorname{deg}\left(v, K_{i}\right) \leq 2$.

Fix two edges $e_{1} \in G\left[S_{1} \backslash E_{1}\right]$ and $e_{2} \in G\left[S_{2} \backslash E_{2}\right]$; such edges exist by Claim 8 , For each $i=1,2$ the link $\mathrm{lk}_{G} e_{3-i}$ lies in $K_{i}$ and its intersection with $K_{i} \backslash E_{i}$ is a collection of at most $3 x$ paths of total length at least $\frac{n}{2}-6 \gamma n$ by Claim 2 , or a sole cycle. Define a segment in $G\left[K_{i}\right]$ as a maximal connected sub-path (or a cycle) of $\mathrm{lk}_{G} e_{3-i}$ which lies in $K_{i} \backslash E_{i}$. (Note that our definition of segments is with respect to fixed edges $e_{1}$ and $e_{2}$.) There are at most $3 x \leq 6 / \gamma$ segments in $K_{i}$. A segment is called long if it has at least $\alpha n$ vertices and short otherwise. The total length of short segments in $K_{i}$ is at most $\frac{6}{\gamma} \cdot \alpha n<0.09 n$; hence the total length of long segments in each $K_{i}$ is at least $0.4 n$.

Claim 10. Let $R_{1}$ and $R_{2}$ be two segments in $K_{1}$ and $K_{2}$, respectively. If for some vertices $x_{1} \in R_{1}, x_{2} \in R_{2}$ we have $x_{1} x_{2} \in E(G)$, then $G\left[R_{1}, R_{2}\right]$ is complete bipartite. 
Proof. If $x_{1}^{\prime}, x_{1}^{\prime \prime}$ are the neighbors of $x_{1}$ in $K_{1}$ and $x_{2}^{\prime}, x_{2}^{\prime \prime}$ are the neighbors of $x_{2}$ in $K_{2}$, then the link $\operatorname{lk}_{G} x_{1} x_{2}$ is a cycle contained in $\left\{x_{1}^{\prime}, x_{1}^{\prime \prime}, x_{2}^{\prime}, x_{2}^{\prime \prime}\right\}$; hence, by Definition 1.4 $\mathrm{b}$ ) it must pass through all those vertices. Therefore $x_{1} x_{2}^{\prime}, x_{1} x_{2}^{\prime \prime}, x_{2} x_{1}^{\prime}, x_{2} x_{1}^{\prime \prime}$ $\in E(G)$. By successively repeating the same argument for the newly forced edges we prove the claim.

Claim 11. If $R_{1}$ and $R_{2}$ are two long segments in $K_{1}$ and $K_{2}$ respectively, then $G\left[R_{1}, R_{2}\right]$ is complete bipartite.

Proof. If not, then, by Claim [10, the bipartite graph $G\left[R_{1}, R_{2}\right]$ does not contain any edges. Then

$$
\bar{e}\left(G\left[K_{1}, K_{2}\right]\right) \geq \bar{e}\left(G\left[R_{1}, R_{2}\right]\right)=\left|R_{1}\right| \cdot\left|R_{2}\right| \geq \frac{\alpha^{2} n^{2}}{2},
$$

a contradiction to (18).

Let $L_{1}$ and $L_{2}$ be the vertex sets of all the long segments in $K_{1}$ and $K_{2}$, respectively. By Claim 11 the graph $G\left[L_{1}, L_{2}\right]$ is complete bipartite. For $i=1,2$ choose edges $\widetilde{e}_{i} \in G\left[L_{i}\right]$ which minimize the quantity

$$
\left|K_{3-i} \backslash V\left(\mathrm{lk}_{G} \widetilde{e}_{i}\right)\right|
$$

and let $C_{i} \subseteq K_{i}$ be the vertex set of the cycle $\operatorname{lk}_{G} \widetilde{e}_{3-i}$.

Claim 12. We have $\left|K_{1} \backslash C_{1}\right|+\left|K_{2} \backslash C_{2}\right| \leq 2$.

Proof. Let $d_{i}=\left|K_{i} \backslash C_{i}\right|$. By the optimality of the choice of $\widetilde{e}_{i}$ we get that the link of every edge in $G\left[L_{i}\right]$ misses at least $d_{3-i}$ vertices of $K_{3-i}$. Since $G\left[L_{1}, L_{2}\right]$ is complete bipartite by Claim[11, those missing edges must contribute to $\bar{e}\left(G\left[L_{i}, K_{3-i} \backslash L_{3-i}\right]\right)$. Recall that $G\left[L_{i}\right]$ is a collection of at most $3 x \leq 6 / \gamma$ vertex-disjoint paths (or a cycle) of total length at least $0.4 n$. We get

$$
\bar{e}\left(G\left[L_{i}, K_{3-i} \backslash L_{3-i}\right]\right) \geq \frac{d_{3-i}}{2}\left(\left|L_{i}\right|-3 x\right) \geq d_{3-i} \cdot 0.19 \cdot n .
$$

The two sets of missing edges we count this way for $i=1,2$ are disjoint. Therefore, using (18)

$$
0.51 n>b \geq \bar{e}\left(G\left[L_{1}, K_{2} \backslash L_{2}\right]\right)+\bar{e}\left(G\left[L_{2}, K_{1} \backslash L_{1}\right]\right) \geq 0.19 n\left(d_{1}+d_{2}\right),
$$

which implies $d_{1}+d_{2}<2.7$. That ends the proof.

The graphs $G\left[C_{1}\right], G\left[C_{2}\right]$ are cycles and the minimizing edges $\widetilde{e}_{i} \in L_{i} \subseteq C_{i}$ satisfy $\operatorname{lk}_{G} \widetilde{e}_{i}=G\left[C_{3-i}\right]$. Together with Claim 12 it shows that $G$ is $t$-joinlike for $t \leq 2$. If $t=0$, then we are done. The case $t=1$ leads to a contradiction as shown in Proposition 4.2. We can therefore assume that $t=2$ and call the two exceptional vertices $q$ and $q^{\prime}$. We can assume without loss of generality that either

$$
K_{1} \backslash C_{1}=\{q\}, \quad K_{2} \backslash C_{2}=\left\{q^{\prime}\right\}
$$

or

$$
K_{1} \backslash C_{1}=\left\{q, q^{\prime}\right\}, \quad K_{2} \backslash C_{2}=\emptyset .
$$

Define the following quantities for $i=1,2$,

$$
\begin{aligned}
d_{i}(q)=\operatorname{deg}\left(q, C_{i}\right) \quad \text { and } & d_{i}\left(q^{\prime}\right)=\operatorname{deg}\left(q^{\prime}, C_{i}\right), \\
e_{i}(q)=e\left(G\left[N_{q} \cap C_{i}\right]\right) \quad \text { and } \quad & e_{i}\left(q^{\prime}\right)=e\left(G\left[N_{q^{\prime}} \cap C_{i}\right]\right) .
\end{aligned}
$$


Note that $e_{i}(q) \leq d_{i}(q)$ and $e_{i}\left(q^{\prime}\right) \leq d_{i}\left(q^{\prime}\right)$ since $G\left[N_{q} \cap C_{i}\right]$ and $G\left[N_{q^{\prime}} \cap C_{i}\right]$ are induced subgraphs of cycles.

If any of the numbers $d_{1}(q), d_{1}\left(q^{\prime}\right), d_{2}(q), d_{2}\left(q^{\prime}\right)$ is at most 2 , then the result follows from Proposition 4.7. We will therefore assume that

$$
\min \left\{d_{1}(q), d_{1}\left(q^{\prime}\right), d_{2}(q), d_{2}\left(q^{\prime}\right)\right\} \geq 3 .
$$

The proof under this assumption splits into the two cases (20) and (21) and is presented in the next section.

\section{Two eXceptional Vertices of LARGE DEGREeS}

In this section we show that each of the cases (20) and (21) from the previous section leads to a contradiction. We use the same notation.

We are going to exploit the fact that the graphs $\operatorname{lk}_{G} q$ and $\mathrm{lk}_{G} q^{\prime}$ are planar. Recall that Euler's formula implies that an $h$-vertex planar graph can have at most $3 h-6$ edges. So, planar graphs are sparse, and a substantial number of edges must be missing between $C_{1}$ and $C_{2}$. A careful edge counting will lead to a contradiction.

We start with an auxiliary claim.

Claim 13. We have an inequality

$$
\bar{e}\left(G\left[N_{q} \cap C_{1}, N_{q} \cap C_{2}\right]\right) \geq d_{1}(q) d_{2}(q)-3 d_{1}(q)-3 d_{2}(q)+e_{1}(q)+e_{2}(q)+6 .
$$

An analogous inequality holds for $q^{\prime}$.

Proof. The graph $G\left[N_{q} \cap\left(C_{1} \cup C_{2}\right)\right]$ is a planar graph with $d_{1}(q)+d_{2}(q)$ vertices and

$$
d_{1}(q) d_{2}(q)-\bar{e}\left(G\left[N_{q} \cap C_{1}, N_{q} \cap C_{2}\right]\right)+e_{1}(q)+e_{2}(q)
$$

edges. The claim now follows from Euler's formula.

From previous estimates we have $\frac{n}{2}-2 \alpha n \leq\left|C_{i}\right| \leq \frac{n}{2}+2 \alpha n$. The next easy statement records the fact that if $q$ is adjacent to most of $C_{i}$, then $\mathrm{lk}_{G} q$ also contains most of the edges from $G\left[C_{i}\right]$.

Claim 14. Suppose $\beta \geq 4 \alpha$. If $d_{i}(q) \geq \frac{n}{2}(1-\beta)$, then $e_{i}(q) \geq \frac{n}{2}(1-5 \beta)$. The same holds for $q^{\prime}$.

Proof. Since $\left|C_{i}\right| \leq \frac{n}{2}+2 \alpha n$ the set $N_{q}$ misses at most

$$
\frac{n}{2}+2 \alpha n-\frac{n}{2}(1-\beta)=n\left(\frac{1}{2} \beta+2 \alpha\right) \leq \beta n
$$

vertices of $C_{i}$. Recall that $G\left[C_{i}\right]$ is a cycle. It follows that at most $2 \beta n$ edges of $G\left[C_{i}\right]$ are not in $\operatorname{lk}_{G} q$. Hence

$$
e_{i}(q) \geq \frac{n}{2}-2 \alpha n-2 \beta n=\frac{n}{2}(1-4 \alpha-4 \beta) \geq \frac{n}{2}(1-5 \beta) .
$$


3.1. The case (20). By Claim 7 we have $d_{1}(q), d_{2}\left(q^{\prime}\right) \leq \frac{4}{\gamma}$. Therefore

$$
\bar{e}\left(G\left[C_{1}, C_{2}\right]\right) \geq \bar{e}\left(G\left[N_{q} \cap C_{1}, N_{q} \cap C_{2}\right]\right)+\bar{e}\left(G\left[N_{q^{\prime}} \cap C_{1}, N_{q^{\prime}} \cap C_{2}\right]\right)-\frac{16}{\gamma^{2}} .
$$

The inequality

$$
\begin{aligned}
\frac{1}{4}\left(n^{2}+2 n+17\right) & <e(G) \leq\left(\frac{n-2}{2}\right)^{2}+n+\operatorname{deg}(q)+\operatorname{deg}\left(q^{\prime}\right)-\bar{e}\left(G\left[C_{1}, C_{2}\right]\right) \\
& \leq \frac{n^{2}}{4}+\operatorname{deg}(q)+\operatorname{deg}\left(q^{\prime}\right) \\
& -\bar{e}\left(G\left[N_{q} \cap C_{1}, N_{q} \cap C_{2}\right]\right)-\bar{e}\left(G\left[N_{q^{\prime}} \cap C_{1}, N_{q^{\prime}} \cap C_{2}\right]\right)+\frac{16}{\gamma^{2}}+1
\end{aligned}
$$

together with Claim 13 and $e_{1}(q), e_{2}\left(q^{\prime}\right) \leq \frac{4}{\gamma}$ gives

(22) $\frac{1}{2} n+\left(d_{1}(q)-4\right)\left(d_{2}(q)-4\right)+\left(d_{1}\left(q^{\prime}\right)-4\right)\left(d_{2}\left(q^{\prime}\right)-4\right)+e_{2}(q)+e_{1}\left(q^{\prime}\right) \leq O(1)$,

where $O(1)$ denotes some universal constant (depending on $\gamma$ ) whose exact value does not matter. Observe that if $d_{1}(q) \geq 4$, then the inequalities $d_{1}(q) \leq \frac{4}{\gamma}$ and $d_{2}(q) \geq 3$ imply $\left(d_{1}(q)-4\right)\left(d_{2}(q)-4\right) \geq-\frac{4}{\gamma}$. A similar observation holds for $q^{\prime}$. Therefore, if $d_{1}(q), d_{2}\left(q^{\prime}\right) \geq 4$, then we get a contradiction because then the left-hand side of (22) is at least $\frac{1}{2} n-\frac{8}{\gamma}$.

Let us then assume that $d_{1}(q)=3$. Then the inequality (22) becomes

$$
\frac{1}{2} n+\left(d_{1}\left(q^{\prime}\right)-4\right)\left(d_{2}\left(q^{\prime}\right)-4\right)+e_{2}(q)+e_{1}\left(q^{\prime}\right) \leq d_{2}(q)+O(1) .
$$

If $d_{2}\left(q^{\prime}\right) \geq 4$, then $\left(d_{1}\left(q^{\prime}\right)-4\right)\left(d_{2}\left(q^{\prime}\right)-4\right) \geq-\frac{4}{\gamma}$, and therefore (23) implies $d_{2}(q) \geq$ $0.49 n$. By Claim 14 we have $e_{2}(q) \geq 0.45 n$, and plugging this back into (23) we get $d_{2}(q) \geq \frac{1}{2} n+0.45 n-O(1) \geq 0.94 n$, which is a contradiction with $d_{2}(q) \leq\left|C_{2}\right| \leq$ $0.51 n$.

We are now left with the case when $d_{1}(q)=d_{2}\left(q^{\prime}\right)=3$ and (23) reduces to

$$
\frac{1}{2} n+e_{2}(q)+e_{1}\left(q^{\prime}\right) \leq d_{2}(q)+d_{1}\left(q^{\prime}\right)+O(1) .
$$

We now need the following claim.

Claim 15. If $v \in C_{2}$ is an isolated vertex of the graph $G\left[N_{q} \cap C_{2}\right]$, then $v q^{\prime} \in E(G)$.

Proof. The cycle $\mathrm{lk}_{G} q v$ is contained in $\left(N_{q} \cap C_{1}\right) \cup\left\{q^{\prime}\right\}$, and since $d_{1}(q)=3$, the latter set has four vertices. By Definition $1.4 \mathrm{~b}) \mathrm{lk}_{G} q v$ must pass through all of them and in particular $q^{\prime} \in N_{v}$.

Because $d_{2}\left(q^{\prime}\right)=3$ the claim implies that $G\left[N_{q} \cap C_{2}\right]$ can have at most three isolated vertices, and therefore $e_{2}(q) \geq \frac{1}{2}\left(d_{2}(q)-3\right)$. By symmetry we get

$$
e_{1}\left(q^{\prime}\right) \geq \frac{1}{2}\left(d_{1}\left(q^{\prime}\right)-3\right)
$$

and (24) implies

$$
n \leq d_{1}\left(q^{\prime}\right)+d_{2}(q)+O(1) .
$$

It follows that $d_{1}\left(q^{\prime}\right), d_{2}(q) \geq 0.48 n$, but then, by Claim 14, $e_{1}\left(q^{\prime}\right), e_{2}(q) \geq 0.4 n$ and going back to the inequality (24) gives a contradiction. 
3.2. The case (21). This time we have $d_{1}(q), d_{1}\left(q^{\prime}\right) \leq \frac{4}{\gamma}$. The missing edges in $G\left[N_{q} \cap C_{1}, N_{q} \cap C_{2}\right]$ and $G\left[N_{q^{\prime}} \cap C_{1}, N_{q^{\prime}} \cap C_{2}\right]$ can have a significant overlap, so we begin by using just the contribution of one of them to obtain a bound. We have $\frac{1}{4}\left(n^{2}+2 n+17\right)<e(G) \leq\left(\frac{n-2}{2}\right)^{2}+n+\operatorname{deg}(q)+\operatorname{deg}\left(q^{\prime}\right)-\bar{e}\left(G\left[N_{q} \cap C_{1}, N_{q} \cap C_{2}\right]\right)$, and plugging in the bound from Claim 13 we obtain

$$
\frac{1}{2} n+\left(d_{1}(q)-4\right)\left(d_{2}(q)-4\right)+e_{2}(q) \leq d_{2}\left(q^{\prime}\right)+O(1) .
$$

In the same way we obtain a symmetric version with $q$ and $q^{\prime}$ interchanged:

$$
\frac{1}{2} n+\left(d_{1}\left(q^{\prime}\right)-4\right)\left(d_{2}\left(q^{\prime}\right)-4\right)+e_{2}\left(q^{\prime}\right) \leq d_{2}(q)+O(1) .
$$

Now suppose that $d_{1}(q) \geq 4$. Then $\left(d_{1}(q)-4\right)\left(d_{2}(q)-4\right) \geq-\frac{4}{\gamma}$, and so (26) implies $d_{2}\left(q^{\prime}\right) \geq 0.49 n$. Therefore, $e_{2}\left(q^{\prime}\right) \geq 0.45 n$ by Claim 14, Then the inequality (27) can be rewritten as

$$
\begin{aligned}
d_{2}(q) & \geq \frac{1}{2} n+\left(d_{1}\left(q^{\prime}\right)-4\right)\left(d_{2}\left(q^{\prime}\right)-4\right)+e_{2}\left(q^{\prime}\right)-O(1) \\
& \geq 0.94 n+\left(d_{1}\left(q^{\prime}\right)-4\right)\left(d_{2}\left(q^{\prime}\right)-4\right) .
\end{aligned}
$$

This inequality can only be satisfied if the last product is negative, which implies $d_{1}\left(q^{\prime}\right)=3$. Using $d_{2}\left(q^{\prime}\right) \leq 0.51 n$ we further obtain

$$
d_{2}(q) \geq 0.94 n-0.51 n=0.43 n .
$$

By Claim 14 we get $e_{2}(q) \geq 0.15 n$; but then (26) gives

$$
d_{2}\left(q^{\prime}\right) \geq \frac{1}{2} n+0.15 n-O(1) \geq 0.64 n,
$$

which is a contradiction.

By symmetry we also arrive at a contradiction assuming that $d_{1}\left(q^{\prime}\right) \geq 4$. It means that we must have $d_{1}(q)=d_{1}\left(q^{\prime}\right)=3$.

We have that $\left|\left(N_{q} \cup N_{q^{\prime}}\right) \cap C_{1}\right| \leq 6$. Consequently, there are only a finite number of possibilities for the graph $G\left[\left(N_{q} \cup N_{q^{\prime}}\right) \cap C_{1}\right]$. We will first show that the actual possibilities for $G\left[\left(N_{q} \cup N_{q^{\prime}}\right) \cap C_{1}\right]$ are even more limited. Call a vertex $v \in C_{1}$ free if $v \notin N_{q} \cup N_{q^{\prime}}$, a q-vertex if $v \in N_{q} \backslash N_{q^{\prime}}$, a $q^{\prime}$-vertex if $v \in N_{q^{\prime}} \backslash N_{q}$, a $q q^{\prime}$-vertex if $v \in N_{q} \cap N_{q^{\prime}}$ and a boundary vertex if $v$ belongs to an edge $e \in G\left[C_{1}\right]$ such that $\mathrm{lk}_{G} e \cap\left\{q, q^{\prime}\right\}=\emptyset$. Observe that each free vertex is also boundary.

Claim 16. The vertices in $C_{1}$ have the following properties:

a) if $v \in C_{1}$ is boundary, then $C_{2} \subseteq N_{v}$,

b) if $v \in C_{1}$ is a $q$-vertex, then at least one of its neighbors in $C_{1}$ is in $N_{q}$,

b') if $v \in C_{1}$ is a $q^{\prime}$-vertex, then at least one of its neighbors in $C_{1}$ is in $N_{q^{\prime}}$,

c) if $v \in C_{1}$ is a $q q^{\prime}$-vertex, then at least one of its neighbors in $C_{1}$ is in $N_{q} \cup N_{q^{\prime}}$,

d) if $e_{1}, e_{2} \in G\left[C_{1}\right]$ are two vertex-disjoint edges, such that $\mathrm{lk}_{G} e_{1}$ contains $q$ but not $q^{\prime}$ and $\operatorname{lk}_{G} e_{2}$ contains $q^{\prime}$ but not $q$, then in at least one of those edges both endpoints are non-boundary,

e) if $v$ is a $q$-vertex and $w$ is a $q^{\prime}$-vertex, then $v w \notin E\left(G\left[C_{1}\right]\right)$. 


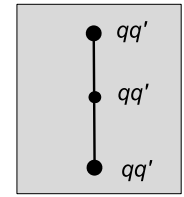

Case A

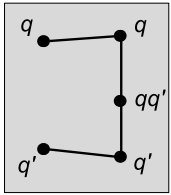

Case $\mathrm{E}$

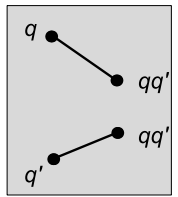

Case B

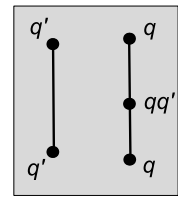

Case F

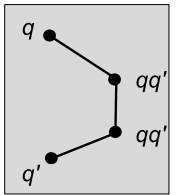

Case C

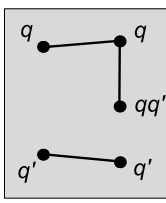

Case G

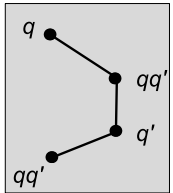

Case D

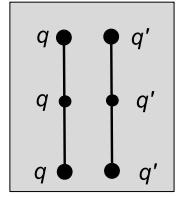

Case $\mathrm{H}$

FIgURE 1. Eight possibilities of the graph $G\left[\left(N_{q} \cup N_{q^{\prime}}\right) \cap C_{1}\right]$ with the types of the vertices (types $q^{-}, q^{\prime}-$, and $q q^{\prime}-$ ).

Proof. a) Consider any edge $e \in G\left[C_{1}\right]$ such that $v \in e$ and $V\left(\operatorname{lk}_{G} e\right) \cap\left\{q, q^{\prime}\right\}=\emptyset$. Then $\mathrm{lk}_{G} e=G\left[C_{2}\right]$, so in particular $C_{2} \subseteq N_{v}$.

b) Let $v^{\prime}, v^{\prime \prime} \in C_{1}$ be the neighbors of $v$. If none of $v^{\prime}, v^{\prime \prime}$ is in $N_{q}$, then all three of $v, v^{\prime}, v^{\prime \prime}$ are boundary, so by a) all are adjacent to the whole $C_{2}$. Pick any vertex $w \in N_{q} \cap C_{2}$ and let $w^{\prime}, w^{\prime \prime}$ be its neighbors in $C_{2}$. Then the link $\operatorname{lk}_{G} v w$ contains the cycle $w^{\prime} v^{\prime} w^{\prime \prime} v^{\prime \prime}$ and the vertex $q$, which is impossible. By symmetry we also get $\left.b^{\prime}\right)$.

c) The proof is the same as b).

d) Suppose the contrary. Let $e_{1}=x x^{\prime}, e_{2}=y y^{\prime}$, where $x^{\prime}$ and $y^{\prime}$ are boundary vertices. By a) $C_{2} \subseteq N_{x^{\prime}}, N_{y^{\prime}}$, therefore

$$
\mathrm{lk}_{G}\left(e_{1}\right)=G\left[\{q\} \cup\left(N_{x} \cap C_{2}\right)\right], \quad \mathrm{lk}_{G}\left(e_{2}\right)=G\left[\left\{q^{\prime}\right\} \cup\left(N_{y} \cap C_{2}\right)\right] .
$$

It follows that $G\left[N_{x} \cap C_{2}\right]$ is a path within $C_{2}$ and $q$ is adjacent only to the endpoints of that path. The same argument for $y$ and $q^{\prime}$ shows that $G\left[N_{y} \cap C_{2}\right]$ is a path with $q^{\prime}$ adjacent only to the endpoints of that path. It follows that, except for up to four special vertices, every vertex in $C_{2}$ is missing an edge to either $q$ or $x$ and it is missing an edge to either $q^{\prime}$ or $y$. Since $x, y, q, q^{\prime}$ are four different vertices this yields at least $2\left(\left|C_{2}\right|-4\right) \approx n$ missing edges from $K_{2}$ to $K_{1}$, contradicting (18).

e) Suppose $v w$ is an edge. Then $v$ and $w$ are both boundary. Let $v^{\prime} v w w^{\prime}$ be the 4-vertex path on the cycle $G\left[C_{1}\right]$. By b) and b') we have $v^{\prime} \in N_{q}$ and $w^{\prime} \in N_{q^{\prime}}$. Then the edges $v v^{\prime}$ and $w w^{\prime}$ contradict $\mathrm{d}$ ).

It turns out that Claim [16 provides us with just enough information to restrict $G\left[\left(N_{q} \cup N_{q^{\prime}}\right) \cap C_{1}\right]$ to just one possibility.

Claim 17. We have $N_{q} \cap C_{1}=N_{q^{\prime}} \cap C_{1}=\left\{v_{1}, v_{2}, v_{3}\right\}$, where $v_{1}, v_{2}, v_{3}$ are three consecutive vertices in $C_{1}$.

Proof. Claim 16 gives us that $G\left[\left(N_{q} \cup N_{q^{\prime}}\right) \cap C_{1}\right]$ is a graph with no cycle, in which every vertex has degree 1 or 2 , and there is no edge from a $q$-vertex to a $q^{\prime}$-vertex. By considering the possible number of $q q^{\prime}$-vertices $(3,2,1$ or 0$)$ and then their degrees, we obtain eight graphs which satisfy the above property, up to exchanging $q$ and $q^{\prime}$. They are shown in Figure 1. The graphs B-H have a pair of edges which violates Claim 16d). That leaves us only with Case A. 
As all the vertices in $C_{1}$ except $v_{2}$ are boundary, we have by Claim 16]) that $C_{2} \subseteq N_{v}$ for each $v \in C_{1} \backslash\left\{v_{2}\right\}$.

Claim 18. There is no edge $e \in G\left[C_{2}\right]$ with $q, q^{\prime} \in \mathrm{lk}_{G} e$.

Proof. If $e$ was such an edge, then $v_{1}$ would be a vertex of degree 3 in $\mathrm{lk}_{G} e$.

Claim 19. We have $\left|N_{q} \cap N_{v_{2}} \cap C_{2}\right| \leq 2$ and $\left|N_{q^{\prime}} \cap N_{v_{2}} \cap C_{2}\right| \leq 2$.

Proof. Any 3 vertices in $N_{q} \cap N_{v_{2}} \cap C_{2}$ together with $\left\{v_{1}, v_{2}, v_{3}\right\}$ would form a $K_{3,3}$ in $\mathrm{lk}_{G} q$, contradicting Definition $[1.4 \mathrm{~d})$.

To complete the proof we consider two cases. First suppose $q q^{\prime} \in E(G)$. Then, we have $\left|N_{q} \cap N_{q^{\prime}} \cap C_{2}\right| \leq 2$. Indeed, otherwise $v_{1}$ would be a vertex of degree at least 3 in $\mathrm{lk}_{G} q q^{\prime}$, a contradiction to Definition 1.4 b). It follows that every vertex of $C_{2}$, except for at most six special ones, is adjacent to at most one element of $\left\{q, q^{\prime}, v_{2}\right\}$, and then there are at least $2\left(\left|C_{2}\right|-6\right) \approx n$ edges missing from $K_{2}$ to $K_{1}$. This contradicts (18).

Now suppose $q q^{\prime} \notin E(G)$. Then $\mathrm{lk}_{G} q v_{3}=G\left[\left\{v_{2}\right\} \cup\left(N_{q} \cap C_{2}\right)\right]$ and $\mathrm{lk}_{G} q^{\prime} v_{3}=$ $G\left[\left\{v_{2}\right\} \cup\left(N_{q^{\prime}} \cap C_{2}\right)\right]$. It means that $G\left[N_{q} \cap C_{2}\right]$ and $G\left[N_{q^{\prime}} \cap C_{2}\right]$ are paths - say $P$ and $P^{\prime}$ - within $C_{2}$. By Claim 18, $P$ and $P^{\prime}$ share at most the endvertices. Moreover, the interior vertices of $P$ and $P^{\prime}$ are not adjacent to $v_{2}$. Consequently, every vertex in $C_{2}$, except for at most four special vertices, is adjacent to at most one element of $\left\{q, q^{\prime}, v_{2}\right\}$. Again, the total number of missing edges from $K_{2}$ to $K_{1}$ is at least $2\left(\left|C_{2}\right|-4\right) \approx n$, contradicting (18).

This ends the consideration of the case (21), thereby completing the proof of Theorem 1.6.

\section{EXACT RESUlts}

In the proof of Theorem 1.6 we used, as black-boxes, two results about the sparseness of certain 1- and 2-joinlike graphs - Propositions 4.2 and 4.7. They will be proved in this section. Unlike previously, when we were free to count edges with an accuracy of $\Theta(n)$, in this part we will need to determine the precise structure of some fascinating graphs and count their edges exactly.

In this section $G$ means any fascinating graph, which will always be 1- or 2joinlike, with $C_{1}, C_{2}$ referring to the cycles from Definition 2.1 and with exceptional vertices called $q$ and $q^{\prime}$. We will frequently use the observation that if $q$ is an exceptional vertex of a $t$-joinlike graph $G$, then $C_{i} \backslash N_{q} \neq \emptyset$ for $i=1,2$.

Proposition 4.1. If $G$ is 1 -joinlike and $q$ is the exceptional vertex, then $\operatorname{deg}\left(q, C_{i}\right)$ $\geq 3$ for $i=1,2$.

Proof. Suppose that $\operatorname{deg}\left(q, C_{1}\right) \leq 2$. If $\operatorname{deg}\left(q, C_{2}\right)=0$, then $\mathrm{lk}_{G} q$ contains at most two vertices, so $G$ fails Definition [1.4 d). Otherwise let $x \in N_{q} \cap C_{2}$ be any vertex with at least one neighbor in $C_{2} \backslash N_{q}$. We see that $\mathrm{lk}_{G} q x$ contains at most three vertices, which is a contradiction.

Proposition 4.2. If $G$ is 1-joinlike, then $e(G) \leq \frac{1}{4}\left(n^{2}+2 n+17\right)$, where $n=|V(G)|$.

Proof. Let $q$ be the exceptional vertex. We will say that a vertex $v \in C_{i}$ is a $q$ vertex if $q v \in E(G)$, a free vertex otherwise and a boundary vertex if it is a $q$-vertex adjacent to a free vertex.

We refer to $C_{1}$ and $C_{2}$ as "sides". 
Claim 20. If $v \in C_{i}$ is free or boundary, then $C_{3-i} \subseteq N_{v}$.

Proof. Indeed, $v$ belongs to an edge $e \in G\left[C_{i}\right]$ with $q \notin \mathrm{lk}_{G} e$ and therefore with $\mathrm{lk}_{G} e=G\left[C_{3-i}\right]$. That means $C_{3-i} \subseteq N_{v}$.

By Proposition 4.1 and because $N_{q} \cap C_{i} \neq C_{i}$ for $i=1,2$, there are at least three $q$-vertices and at least two boundary vertices on each side. If there were three boundary vertices in, say, $C_{1}$, then the graph formed by those three vertices in $C_{1}$ and any three neighbors of $q$ in $C_{2}$ would form, by Claim 20, a $K_{3,3}$ in $\operatorname{lk}_{G} q$, which is impossible. That implies there are exactly two boundary vertices on each side. In other words each $N_{q} \cap C_{i}$ induces a path inside $C_{i}$ of some length $a_{i} \geq 3$ for $i=1,2$.

If $u \in C_{1}$ and $w \in C_{2}$ are $q$-vertices which are not boundary and $u w \in E(G)$, then by Claim 20 there is a $K_{3,3}$ in $\mathrm{lk}_{G} q$ formed by $u, w$ and the two boundary vertices on each side. This means $u w \notin E(G)$ for such $u, w$.

We now know the exact structure of $G$ and we can compute its number of edges. Denoting $c_{i}=\left|C_{i}\right|$ and using $n=c_{1}+c_{2}+1$ we have

$$
\begin{aligned}
e(G) & =c_{1} c_{2}+c_{1}+c_{2}+a_{1}+a_{2}-\left(a_{1}-2\right)\left(a_{2}-2\right) \\
& =\frac{1}{4}\left(n^{2}+2 n+17\right)-\frac{1}{4}\left(c_{1}-c_{2}\right)^{2}-\left(a_{1}-3\right)\left(a_{2}-3\right) \leq \frac{1}{4}\left(n^{2}+2 n+17\right) .
\end{aligned}
$$

The second part of the analysis in this section deals with 2-joinlike graphs. We start off with a counterpart of Proposition 4.1

Proposition 4.3. If $G$ is 2 -joinlike and $q$ is any exceptional vertex, then $\operatorname{deg}\left(q, C_{i}\right)$ $\geq 2$ for $i=1,2$.

Proof. Suppose that $\operatorname{deg}\left(q, C_{1}\right) \leq 1$. If $\operatorname{deg}\left(q, C_{2}\right)=0$, then $\mathrm{lk}_{G} q$ contains at most two vertices, so $G$ fails Definition $1.4 \mathrm{~d}$ ). Otherwise let $x \in N_{q} \cap C_{2}$ be any vertex with at least one neighbor in $C_{2} \backslash N_{q}$. We see that $\operatorname{lk}_{G} q x$ contains at most three vertices, which is a contradiction.

We shall later need the following simple inequality.

Lemma 4.4. If $n=k+l+2$, then

$$
k l+2 k+l+6 \leq \frac{1}{4}\left(n^{2}+2 n+17\right) .
$$

Proof. One checks that

$$
k l+2 k+l+6=\frac{1}{4}\left(n^{2}+2 n+17\right)-\frac{1}{4}(l-k+1)^{2} .
$$

Proposition 4.7 below is a combination of a case distinction captured by Proposition 4.5 and Proposition 4.6.

Proposition 4.5. If $G$ is 2-joinlike with exceptional vertices $\left\{q, q^{\prime}\right\}$ such that $\operatorname{deg}\left(q, C_{1}\right)=2$ and the two vertices of $N_{q} \cap C_{1}$ are adjacent, then $e(G) \leq$ $\frac{1}{4}\left(n^{2}+2 n+17\right)$, where $n=|V(G)|$. 
Proof. Let $N_{q} \cap C_{1}=\{u, v\}$. Let $x, x^{\prime} \in C_{2}$ be neighbors such that $q x \in E(G)$, $q x^{\prime} \notin E(G)$ and let $y$ be the other neighbor of $x$ in $C_{2}$ (their existence is guaranteed by Proposition 4.3 and the fact that $\left.N_{q} \cap C_{2} \neq C_{2}\right)$. Then $V\left(\operatorname{lk}_{G} q x\right) \subseteq\left\{u, v, q^{\prime}, y\right\}$, and since $u v \in E(G)$ we can assume that $\operatorname{lk}_{G} q x$ is the cycle $v u y q^{\prime}$ (this is the unique possibility up to the order of $u, v)$. In particular, $q q^{\prime}, q^{\prime} v \in E(G)$ and $q^{\prime} u \notin E(G)$.

If $u^{\prime} \neq v$ is the other neighbor of $u$ in $C_{1}$, then $\operatorname{lk}_{G} u u^{\prime}$ contains neither $q$ nor $q^{\prime}$, so it must be all of $C_{2}$. In particular, $C_{2} \subseteq N_{u}$. It means that $\mathrm{lk}_{G} u q=$ $G\left[\{v\} \cup\left(N_{q} \cap C_{2}\right)\right]$, so $G\left[N_{q} \cap C_{2}\right]$ is a path of length at least 3 within $C_{2}$, whose both endpoints, call them $v_{1}, v_{2}$, are connected to $v$, while the interior vertices of the path are not connected to $v$. (In fact $x$ from the previous paragraph is one of the $v_{i}$.) Let $a=\left|N_{q} \cap C_{2}\right|$ be the length of this path.

The link of every edge in $G\left[N_{q} \cap C_{2}\right]$ contains $u$ and $q$, so to be a cycle it must also contain $q^{\prime}$. It follows that $N_{q^{\prime}} \cap C_{2} \supseteq N_{q} \cap C_{2}$.

Let $t \neq u$ be the other neighbor of $v$ in $C_{1}$. We now focus on the link $\operatorname{lk}_{G} q^{\prime} v$. It contains the path $v_{1} q v_{2}$. As we shall see, the case $t \notin \mathrm{lk}_{G} q^{\prime} v$ will lead to a contradiction.

Claim 21. If $t \notin \mathrm{lk}_{G} q^{\prime} v$, then $\mathrm{lk}_{G} q^{\prime} v$ must contain, apart from $v_{1}, q$ and $v_{2}$, all the vertices in $C_{2} \backslash N_{q}$.

Proof. The link $\operatorname{lk}_{G} q^{\prime} v$ is a cycle which passes through $v_{1} q v_{2}$. The only possible route for this cycle which does not take it outside $\operatorname{lk}_{G} v$ and avoids $t$ and $u$ is to continue from $v_{2}$ back to $v_{1}$ in $C_{2}$, i.e., follow the path $G\left[C_{2} \backslash N_{q}\right]$.

However, the above would imply $C_{2} \backslash N_{q} \subseteq N_{q^{\prime}}$. Put together with the previously established $N_{q^{\prime}} \cap C_{2} \supseteq N_{q} \cap C_{2}$ we would get $C_{2} \subseteq N_{q^{\prime}}$, a contradiction. This means that $t \in \mathrm{lk}_{G} q^{\prime} v$, i.e. $q^{\prime} t \in E(G)$.

Consider any vertex $x \in\left(C_{1} \cap N_{q^{\prime}}\right) \backslash\{v\}$ which has at least one neighbor $\tilde{x}$ in $C_{1} \backslash N_{q^{\prime}}$. By the fact that $q^{\prime} u \notin E(G)$ such a vertex must exist. The link $\operatorname{lk}_{G} x \tilde{x}$ is a cycle which does not touch $C_{1} \cup\left\{q, q^{\prime}\right\}$. Consequently, $\operatorname{lk}_{G} x \tilde{x}=G\left[C_{2}\right]$ and, in particular, $C_{2} \subseteq N_{x}$. The link $\operatorname{lk}_{G} x q^{\prime}$ consists of one vertex in $C_{1}$ and of the whole $N_{q^{\prime}} \cap C_{2}$. We get that $G\left[N_{q^{\prime}} \cap C_{2}\right]$ is a path within $C_{2}$, containing $N_{q} \cap C_{2}$. Let $w_{1}, w_{2}$ be the endpoints and let $b=\left|N_{q^{\prime}} \cap C_{2}\right|$. Assume that $v_{1}$ is between $w_{1}$ and $v_{2}$ on this path (possibly $w_{1}=v_{1}$ or $w_{2}=v_{2}$ ).

For every edge $e$ in $G\left[\left(C_{2} \backslash N_{q^{\prime}}\right) \cup\left\{w_{1}, w_{2}\right\}\right]$ we have $\mathrm{lk}_{G} e=G\left[C_{1}\right]$. As $C_{2} \cap N_{q^{\prime}}$ induces a path with endvertices $w_{1}$ and $w_{2}$ and $G\left[C_{2}\right]$ is a cycle, we must have that $G\left[\left(C_{2} \backslash N_{q^{\prime}}\right) \cup\left\{w_{1}, w_{2}\right\}\right]$ is a path; in particular, this graph contains no isolated vertices. It follows that for every vertex $x \in\left(C_{2} \backslash N_{q^{\prime}}\right) \cup\left\{w_{1}, w_{2}\right\}$ we have $C_{1} \subseteq N_{x}$. Now consider the link $\operatorname{lk}_{G} q^{\prime} v$. It contains the vertices $q, t, v_{1}, v_{2}, w_{1}, w_{2}$, with paths $v_{1} q v_{2}$ and $w_{1} t w_{2}$. This is only possible if $v$ is adjacent to all of $\left(N_{q^{\prime}} \backslash N_{q}\right) \cap C_{2}$ while $t$ is not adjacent to any vertex of $\left(\left(\left(N_{q^{\prime}} \backslash N_{q}\right) \cap C_{2}\right) \cup\left\{v_{1}, v_{2}\right\}\right) \backslash\left\{w_{1}, w_{2}\right\}$.

Let $\left|C_{1}\right|=k,\left|C_{2}\right|=l$, with $n=k+l+2$. The remaining part of the proof splits into two cases. First we assume that $t$ is non-adjacent to all of $\left(N_{q} \cap C_{2}\right) \backslash\left\{v_{1}, v_{2}\right\}$. In that case $t$ is non-adjacent to $b-2$ vertices of $C_{2}, v$ is non-adjacent to $a-2$ vertices and using a bound $\operatorname{deg}\left(q^{\prime}, C_{1}\right) \leq k-1$ we get

$$
\begin{aligned}
e(G) & \leq k l+k+l+(a+2)+(b+k-1)+1-(a-2)-(b-2) \\
& =k l+2 k+l+6
\end{aligned}
$$

so the conclusion follows from Lemma 4.4. 
Next suppose that $t$ has a neighbor $y$ in $\left(N_{q} \cap C_{2}\right) \backslash\left\{v_{1}, v_{2}\right\}$ and let $s \neq v$ be the other neighbor of $t$ in $C_{1}$. The link $\mathrm{lk}_{G} q^{\prime} t$ contains $v, w_{1}, w_{2}, y$ and possibly $s$ with edges $w_{1} v w_{2}$, and apart from $v$ and $s$ it is contained in $N_{q^{\prime}} \cap C_{2}$. Any cycle with that property must contain an edge $e \in G\left[N_{q} \cap C_{2}\right]$, and it follows that there exists an edge $e \in G\left[N_{t} \cap N_{q} \cap C_{2}\right]$. But $\operatorname{lk}_{G} e$ is a cycle passing through $u q q^{\prime} t$ and not through $v$, therefore necessarily going through all of $C_{1} \backslash\{v\}$. In particular, $N_{q^{\prime}} \cap C_{1}=\{v, t\}$, and so $s \notin \mathrm{lk}_{G} q^{\prime} t$. It means that $\operatorname{lk}_{G} q^{\prime} t=G\left[\{v\} \cup\left(N_{q^{\prime}} \cap C_{2}\right)\right]$ which, by the restrictions on $N_{t}$, implies $v_{1}=w_{1}, v_{2}=w_{2}, a=b$ and $C_{2} \subseteq N_{t}$. This determines the graph $G$, and we obtain

$$
\begin{aligned}
e(G) & =k l+k+l+(a+2)+(a+2)+1-(a-2) \\
& =k l+k+l+a+7 \\
& =\frac{1}{4}\left(n^{2}+2 n+17\right)-\frac{1}{4}(k-l+1)^{2}-(l-1-a) \leq \frac{1}{4}\left(n^{2}+2 n+17\right)
\end{aligned}
$$

because $a \leq l-1$.

Proposition 4.6. If $G$ is 2-joinlike with exceptional vertices $\left\{q, q^{\prime}\right\}$ such that $\operatorname{deg}\left(q, C_{1}\right)=2$ and the two vertices of $N_{q} \cap C_{1}$ are not adjacent, then $e(G) \leq$ $\frac{1}{4}\left(n^{2}+2 n+17\right)$ where $n=|V(G)|$.

Proof. The proof uses similar techniques as the proof of Proposition 4.5. Set $N_{q} \cap$ $C_{1}=\{u, v\}$.

Let $x \in C_{2}$ be any vertex with $q x \in E(G)$ and such that $x$ has a neighbor $x^{\prime} \in C_{2}$ with $q x^{\prime} \notin E(G)$. Let $y$ be the other neighbor of $x$ in $C_{2}$. We have $V\left(\operatorname{lk}_{G} q x\right) \subseteq\left\{u, v, q^{\prime}, y\right\}$, with $u$ and $v$ being independent. It follows that $\operatorname{lk}_{G} q x$ is the cycle $u q^{\prime} v y$; in particular, $q^{\prime} u, q^{\prime} v, u x, v x, q^{\prime} x \in E(G)$ and $q q^{\prime} \in E(G)$.

It follows that the number of vertices $x \in C_{2}$ with the property described in the previous paragraph is at most 2 . Indeed, we proved that every such vertex is adjacent to $u, v, q^{\prime}$, and the claim follows since $\mathrm{lk}_{G} q$ is $K_{3,3}$-free. It means that $G\left[N_{q} \cap C_{2}\right]$ is a path within $C_{2}$ of length $a=\left|N_{q} \cap C_{2}\right|$. Moreover, if $v_{1}, v_{2} \in C_{2}$ are the endpoints of that path, then $q^{\prime} v_{j}, u v_{j}, v v_{j} \in E(G)$ for $j=1,2$. It follows that $a \geq 3$, as otherwise $\operatorname{lk}_{G} q u$ would contain a triangle $q^{\prime} v_{1} v_{2}$.

The link $\mathrm{lk}_{G} q u$ contains $q^{\prime}, v_{1}, v_{2}$ and no vertex in $C_{1}$, so it must be $G\left[\left\{q^{\prime}\right\} \cup\left(N_{q} \cap C_{2}\right)\right]$. That, and the same argument for $\operatorname{lk}_{G} q v$, mean that $N_{q} \cap C_{2} \subseteq$ $N_{u}, N_{v}$ and that $q^{\prime}$ is non-adjacent to vertices in $\left(N_{q} \cap C_{2}\right) \backslash\left\{v_{1}, v_{2}\right\}$.

We will now prove the following claim.

Claim 22. Suppose $x \in C_{1} \backslash\{u, v\}$ and $y \in\left(N_{q} \cap C_{2}\right) \backslash\left\{v_{1}, v_{2}\right\}$. Let $x^{\prime}, x^{\prime \prime}$ be the neighbors of $x$ in $C_{1}$, and let $y^{\prime}, y^{\prime \prime}$ be the neighbors of $y$ in $C_{2}$. If $x y \in E(G)$, then $x y^{\prime}, x y^{\prime \prime}, x^{\prime} y, x^{\prime \prime} y \in E(G)$.

Proof. The link $\mathrm{lk}_{G} x y$ contains neither $q$ nor $q^{\prime}$. Hence it must be contained in $\left\{x^{\prime}, x^{\prime \prime}, y^{\prime}, y^{\prime \prime}\right\}$, and it follows that these four vertices must form a 4-cycle with $x$ and $y$ adjacent to all of them.

The vertices $u, v$ divide $G\left[C_{1}\right]$ into two paths which we call $P_{1}, P_{2}$, so that there is a partition $C_{1}=P_{1} \sqcup P_{2} \sqcup\{u, v\}$. We also write $\overline{P_{j}}=P_{j} \cup\{u, v\}$ for $j=1,2$ for the "closures" of those paths. Claim 22 implies that for $j=1,2$ the bipartite graph $G\left[P_{j},\left(N_{q} \cap C_{2}\right) \backslash\left\{v_{1}, v_{2}\right\}\right]$ is either edgeless or complete bipartite. Suppose first that both of these graphs are complete. Take any edge $e$ in $G\left[N_{q} \cap C_{2}\right]$. As $a \geq 3$, such an edge exists. The above then gives that $\mathrm{lk}_{G} e$ contains all of $C_{1}$, and 
$q$, a contradiction. Suppose next that both of these graphs are empty. Taking any edge $e$ in $G\left[N_{q} \cap C_{2}\right]$ we observe that $\operatorname{lk}_{G} e$ spans at most three vertices $\{q, u, v\}$, again a contradiction. We can therefore assume that $G\left[\overline{P_{1}}, N_{q} \cap C_{2}\right]$ is complete bipartite and $G\left[P_{2},\left(N_{q} \cap C_{2}\right) \backslash\left\{v_{1}, v_{2}\right\}\right]$ has no edges.

For every edge $f \in G\left[\overline{P_{2}}\right]$ the link $\mathrm{lk}_{G} f$ misses $q$ and $N_{q} \backslash\left\{v_{1}, v_{2}\right\}$; hence it must contain $q^{\prime}$. We therefore have that

$$
\overline{P_{2}} \subseteq N_{q^{\prime}} .
$$

The rest of the proof depends on whether $N_{q^{\prime}} \cap P_{1}$ is empty.

First suppose that $q^{\prime}$ is adjacent to some vertex of $P_{1}$. Recalling that $N_{q^{\prime}} \cap$ $C_{1} \neq C_{1}$ and combining this with (28) we have $N_{q^{\prime}} \cap P_{1} \neq P_{1}$. We can find $t \in P_{1}$ with neighbors $t^{\prime}, t^{\prime \prime} \in \overline{P_{1}}$ such that $t q^{\prime} \in E(G)$ and $t^{\prime} q^{\prime} \notin E(G)$. Since $\mathrm{lk}_{G} t t^{\prime}$ contains neither $q$ nor $q^{\prime}$ it must be all of $C_{2}$; hence $C_{2} \subseteq N_{t}$. We then have $\operatorname{lk}_{G} q^{\prime} t=G\left[\left\{t^{\prime \prime}\right\} \cup\left(N_{q^{\prime}} \cap C_{2}\right)\right]$, so $N_{q^{\prime}} \cap C_{2}$ induces a path within $C_{2}$ and $t^{\prime \prime}$ is not adjacent to its internal vertices. Since $v_{1}, v_{2} \in N_{q^{\prime}} \cap C_{2}$ we obtain that $N_{q^{\prime}} \cap C_{2}=\left(C_{2} \backslash N_{q}\right) \cup\left\{v_{1}, v_{2}\right\}$.

Let $\left|C_{1}\right|=k,\left|C_{2}\right|=l$. Subtracting the edges we lose from $P_{2}$ to $\left(N_{q} \cap C_{2}\right) \backslash$ $\left\{v_{1}, v_{2}\right\}$ and from $t^{\prime \prime} \in \overline{P_{1}}$ to $C_{2} \backslash N_{q}$ and using $\operatorname{deg}\left(q^{\prime}, C_{1}\right) \leq k-1,\left|P_{2}\right| \geq 1$ and $a \geq 3$, we get

$$
\begin{aligned}
e(G) & \leq k l+k+l+(a+2)+(l-a+2+k-1)+1-\left|P_{2}\right|(a-2)-(l-a) \\
& \leq k l+2 k+l+6 .
\end{aligned}
$$

Next consider the case $N_{q^{\prime}} \cap P_{1}=\emptyset$. By the usual argument we have $C_{2} \subseteq$ $N_{u}, N_{v}$. Let $s \in P_{2}$ be the neighbor of $v$. Then $\operatorname{lk}_{G} q^{\prime} v=G\left[\{s, q\} \cup\left(N_{q^{\prime}} \cap C_{2}\right)\right]$ and it contains the edges $v_{1} q v_{2}$. It follows that there are vertices $w_{1}, w_{2} \in C_{2}$ such that $G\left[N_{q^{\prime}} \cap C_{2}\right]$ has two parts, stretching from $v_{1}$ to $w_{1}$ and from $v_{2}$ to $w_{2}$ (possibly $w_{1}=v_{1}$ or $\left.w_{2}=v_{2}\right)$. Moreover, looking at $\operatorname{lk}_{G} q^{\prime} v$ we see that $s w_{1}, s w_{2} \in E(G)$ but $s$ is not adjacent to the vertices in $\left(N_{q^{\prime}} \cap C_{2}\right) \backslash\left\{w_{1}, w_{2}\right\}$.

Let $b=\left|N_{q^{\prime}} \cap C_{2}\right|$. Counting the missing edges from $P_{2}$ to $\left(N_{q} \cap C_{2}\right) \backslash\left\{v_{1}, v_{2}\right\}$ and the disjoint set of missing edges from $s$ to $\left(N_{q^{\prime}} \cap C_{2}\right) \backslash\left\{w_{1}, w_{2}\right\}$, we have

$$
\begin{aligned}
e(G) & \leq k l+k+l+(a+2)+(b+k-1)+1-\left|P_{2}\right|(a-2)-(b-2) \\
& \leq k l+2 k+l+6 .
\end{aligned}
$$

An application of Lemma 4.4 completes the proof.

Putting the above results (Propositions 4.3, 4.5] and 4.6) together we get the main result of this section concerning 2-joinlike graphs.

Proposition 4.7. If $G$ is 2 -joinlike with exceptional vertices $\left\{q, q^{\prime}\right\}$ and $\operatorname{deg}\left(q, C_{1}\right)$ $\leq 2$, then $e(G) \leq \frac{1}{4}\left(n^{2}+2 n+17\right)$ where $n=|V(G)|$.

\section{Closing remarks}

A careful analysis of the proofs in Section 4 reveals two families of fascinating graphs which satisfy the equality $m=\frac{1}{4}\left(n^{2}+2 n+17\right)$ for $n \geq n_{0}$. They appear in Proposition 4.2 and Proposition 4.5] see Figure 2. This proves the claim made in Remark 1.7 we omit the details.

Let us finish by stating a generalization of Theorem 1.2 to higher dimensions. 


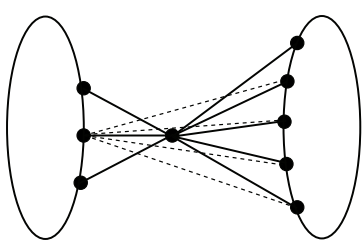

a)

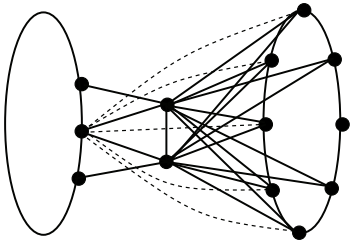

b)

Figure 2. The 1-skeleta of two triangulations of $S^{3}$ with $f_{1}=$ $\frac{1}{4}\left(f_{0}^{2}+2 f_{0}+17\right)$. Starting from the join of two cycles, remove the dashed edges and add the exceptional point(s) with the solid edges. In a) $\left|C_{1}\right|=\left|C_{2}\right|$ and $\operatorname{deg}\left(q, C_{1}\right)=3$. In b) $\left|C_{2}\right|=\left|C_{1}\right|+1$, $\operatorname{deg}\left(q, C_{1}\right)=\operatorname{deg}\left(q^{\prime}, C_{1}\right)=2$ and $\operatorname{deg}\left(q, C_{2}\right)=\operatorname{deg}\left(q^{\prime}, C_{2}\right)=\left|C_{2}\right|-1$.

Conjecture 5.1. For every $s \geq 2$ there exists a number $n_{0}=n_{0}(s)$ such that the following holds. If $M$ is a closed flag $(2 s-1)$-manifold or a flag $(2 s-1)$-GHS with $f_{0} \geq n_{0}$ vertices and $f_{1}$ edges, then

$$
f_{1} \leq f_{0}^{2} \cdot \frac{s-1}{2 s}+f_{0} .
$$

Moreover, if $M$ satisfies

$$
f_{1}>f_{0}^{2} \cdot \frac{s-1}{2 s}+f_{0} \cdot \frac{s-1}{s}+\frac{7 s+3}{2 s},
$$

then $M$ is a join of $s$ polygons, in particular it is homeomorphic to $S^{2 s-1}$.

The maximal value in (29) is achieved by the balanced join of $s$ cycles of lengths $f_{0} / s$. The expression in (30) is the number of edges in the single edge-subdivision of such a join.

Let us sketch how one might prove this conjecture (the details will appear elsewhere). Fix $s \geq 2$ and denote $n=f_{0}$. First of all, $M$ is Eulerian and the "middle" Dehn-Sommerville equation $h_{s-1}=h_{s+1}$ can be rewritten in the form

$$
f_{s}=s f_{s-1}+a_{2} f_{s-2}+\cdots+a_{s} f_{0}
$$

for some coefficients $a_{i}$ depending only on $s$. It follows that the number of $(s+1)$ cliques in the 1-skeleton $G=M^{(1)}$ is only $O\left(n^{s}\right)$. However, the number of edges in $G$ is above the Turán bound for a complete, balanced $s$-partite graph, which is the maximizer of the number of edges among $K_{s+1}$-free graphs. By an application of the stability method we get that $G$ looks very similar to $K_{\ell, \ell, \ldots, \ell}$, where $\ell=n / s$. Next, as in the case of fascinating graphs, we see that in $G$ the link of every $(2 s-1-j)$ clique is a triangulation of $S^{j}$ for $j=0,1,2$ (or for all $0 \leq j \leq 2 s-2$ if $M$ is a manifold), and one can try to exploit those conditions to rigidify the structure of $G$.

\section{ACKNOWLEDGEMENT}

We thank Anna Adamaszek, Martina Kubitzke and Eran Nevo for discussions on this and related topics and the anonymous referee for a number of remarks, including a correction to the proof of Claim 17 


\section{REFERENCES}

[CD95] Ruth Charney and Michael Davis, The Euler characteristic of a nonpositively curved, piecewise Euclidean manifold, Pacific J. Math. 171 (1995), no. 1, 117-137. MR.1362980 (96k:53066)

[ES83] Paul Erdős and Miklós Simonovits, Supersaturated graphs and hypergraphs, Combinatorica 3 (1983), no. 2, 181-192, DOI 10.1007/BF02579292. MR726456 (85e:05125)

[Gal05] Światosław R. Gal, Real root conjecture fails for five-and higher-dimensional spheres, Discrete Comput. Geom. 34 (2005), no. 2, 269-284, DOI 10.1007/s00454-005-1171-5. MR2155722(2006c:52019)

[KS96] J. Komlós and M. Simonovits, Szemerédi's regularity lemma and its applications in graph theory, Combinatorics, Paul Erdős is eighty, Vol. 2 (Keszthely, 1993), Bolyai Soc. Math. Stud., vol. 2, János Bolyai Math. Soc., Budapest, 1996, pp. 295-352. MR.1395865 (97d:05172)

[McM71] P. McMullen, The numbers of faces of simplicial polytopes, Israel J. Math. 9 (1971), 559-570. MR0278183 (43 \#3914)

[MN] S. Murai and E. Nevo, The flag f-vectors of Gorenstein* order complexes of dimension 3, Proc. Amer. Math. Soc. 142 (2014), No. 5, 1527-1538. MR3168460

[NP11] Eran Nevo and T. Kyle Petersen, On $\gamma$-vectors satisfying the Kruskal-Katona inequalities, Discrete Comput. Geom. 45 (2011), no. 3, 503-521, DOI 10.1007/s00454-010-92436. MR2770549(2012d:52026)

[NPT11] Eran Nevo, T. Kyle Petersen, and Bridget Eileen Tenner, The $\gamma$-vector of a barycentric subdivision, J. Combin. Theory Ser. A 118 (2011), no. 4, 1364-1380, DOI 10.1016/j.jcta.2011.01.001. MR2755087 (2012h:52041)

[Sim68] M. Simonovits, A method for solving extremal problems in graph theory, stability problems, Theory of Graphs (Proc. Colloq., Tihany, 1966), Academic Press, New York, 1968, pp. 279-319. MR0233735 (38 \#2056)

[Sze78] Endre Szemerédi, Regular partitions of graphs (English, with French summary), Problèmes combinatoires et théorie des graphes (Colloq. Internat. CNRS, Univ. Orsay, Orsay, 1976), Colloq. Internat. CNRS, vol. 260, CNRS, Paris, 1978, pp. 399-401. MR.540024(81i:05095)

Fachbereich Mathematik, Universität Bremen, Bibliothekstr. 1, 28359 Bremen, GerMANY

E-mail address: aszek@mimuw.edu.pl

Current address: Max Planck Institute for Informatics, Campus E1 4, 66123 Saarbrücken, Germany

Mathematics Institute and DimAP, University of Warwick, Coventry, CV4 7AL, UNITED KINGDOM

E-mail address: honzahladky@gmail.com 